\title{
Conjugating precision and acquisition time in a Doppler broadening regime by interleaved frequency-agile rapid-scanning cavity ring-down spectroscopy
}

Riccardo Gotti, Davide Gatti, Piotr Masłowski, Marco Lamperti, Michele Belmonte, Paolo Laporta, and Marco Marangoni

Citation: The Journal of Chemical Physics 147, 134201 (2017); doi: 10.1063/1.4999056

View online: https://doi.org/10.1063/1.4999056

View Table of Contents: http://aip.scitation.org/toc/jcp/147/13

Published by the American Institute of Physics

\section{Articles you may be interested in}

Experimental and theoretical studies of the reactions of ground-state sulfur atoms with hydrogen and deuterium

The Journal of Chemical Physics 147, 134302 (2017); 10.1063/1.4991418

Detection and structural characterization of nitrosamide $\mathrm{H}_{2} \mathrm{NNO}$ : A central intermediate in de $\mathrm{NO}_{\mathrm{x}}$ processes The Journal of Chemical Physics 147, 134301 (2017); 10.1063/1.4992097

Communication: A hydrogen-bonded difluorocarbene complex: Ab initio and matrix isolation study The Journal of Chemical Physics 147, 131102 (2017); 10.1063/1.4999772

Communication: Molecular near-infrared transitions determined with sub-kHz accuracy

The Journal of Chemical Physics 147, 091103 (2017); 10.1063/1.4998763

Communication: Broad manifold of excitonic states in light-harvesting complex 1 promotes efficient unidirectional energy transfer in vivo

The Journal of Chemical Physics 147, 131101 (2017); 10.1063/1.4999057

Support for the existence of invertible maps between electronic densities and non-analytic 1-body external potentials in non-relativistic time-dependent quantum mechanics

The Journal of Chemical Physics 147, 134110 (2017); 10.1063/1.4991870

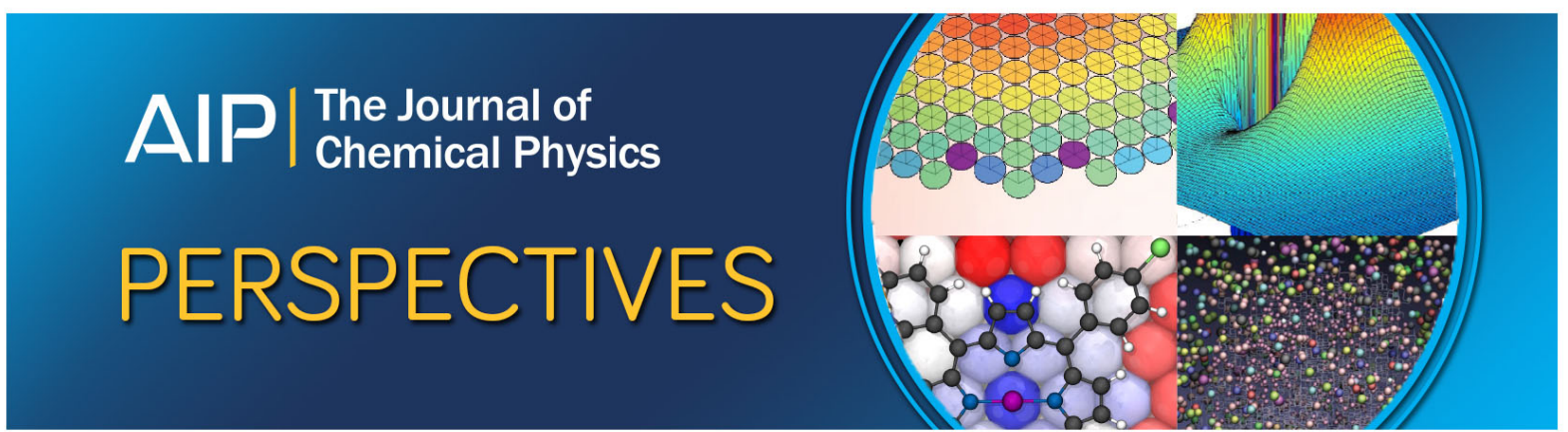




\title{
Conjugating precision and acquisition time in a Doppler broadening regime by interleaved frequency-agile rapid-scanning cavity ring-down spectroscopy
}

\author{
Riccardo Gotti, ${ }^{1}$ Davide Gatti, ${ }^{1}$ Piotr Masłowski, ${ }^{2}$ Marco Lamperti, ${ }^{1}$ Michele Belmonte, ${ }^{3}$ \\ Paolo Laporta, ${ }^{1}$ and Marco Marangoni ${ }^{1}$ \\ ${ }^{1}$ Dipartimento di Fisica-Politecnico di Milano and IFN-CNR, Via Gaetano Previati 1/C, 23900 Lecco, Italy \\ ${ }^{2}$ Institute of Physics, Faculty of Physics, Astronomy and Informatics, Nicolaus Copernicus University, \\ Grudziadzka 5, 87-100 Torun, Poland \\ ${ }^{3}$ Oclaro, Inc., Via F. Fellini, 4, 20097 San Donato Milanese, Italy
}

(Received 4 August 2017; accepted 19 September 2017; published online 5 October 2017)

\begin{abstract}
We propose a novel approach to cavity-ring-down-spectroscopy (CRDS) in which spectra acquired with a frequency-agile rapid-scanning (FARS) scheme, i.e., with a laser sideband stepped across the modes of a high-finesse cavity, are interleaved with one another by a sub-millisecond readjustment of the cavity length. This brings to time acquisitions below $20 \mathrm{~s}$ for few-GHz-wide spectra composed of a very high number of spectral points, typically 3200 . Thanks to the signal-to-noise ratio easily in excess of 10000 , each FARS-CRDS spectrum is shown to be sufficient to determine the line-centre frequency of a Doppler broadened line with a precision of 2 parts over $10^{11}$, thus very close to that of sub-Doppler regimes and in a few-seconds time scale. The referencing of the probe laser to a frequency comb provides absolute accuracy and long-term reproducibility to the spectrometer and makes it a powerful tool for precision spectroscopy and line-shape analysis. The experimental approach is discussed in detail together with experimental precision and accuracy tests on the $(30012) \leftarrow(00001)$ P12e line of $\mathrm{CO}_{2}$ at $\sim 1.57 \mu \mathrm{m}$. Published by AIP Publishing. https://doi.org/10.1063/1.4999056
\end{abstract}

\section{INTRODUCTION}

The progress of optical spectrometers over the past decade has been fuelled by several technological advancements, such as optical frequency combs and high-finesse optical cavities. These have opened up the opportunity for particularly ambitious spectroscopic experiments. Among them, the accurate measurement of the absorption frequencies of light diatomic molecules such as $\mathrm{H}_{2}$ and $\mathrm{D}_{2}$ is recognized as a compelling way to test quantum-electro-dynamic (QED) models for molecules at the part-per-billion level. ${ }^{1}$ Another demanding example is Doppler Broadening Thermometry (DBT), where thermodynamic quantities of a gas at thermodynamic equilibrium are inferred from a Dopplerwidth measurement down to the ppm level. ${ }^{2,4}$ In these and many other cases, the achievement of the scientific goal is strictly related to the adoption of an adequate technological approach.

High-finesse optical cavities are a recognized tool to increase the sensitivity of a spectrometer, enabling it to address either weak transitions (such as quadrupole transitions in QED experiments ${ }^{3,5}$ ) or to explore low-pressure spectroscopy (as it is required, e.g., for sub-Doppler spectroscopy ${ }^{6}$ or for some DBT experiments ${ }^{7}$ ). The reduction of statistical uncertainties and the corresponding improvement of the precision of the measurement require the sensitivity of the spectrometer to be conjugated with a fast acquisition time. In an effort of counteracting the fundamental trade-off between sensitivity and speed, in other words of minimizing the noise-equivalent-absorption (NEA) of the spectrometer, a certain number of approaches have been successfully deployed in cavity-ring-down-spectroscopy (CRDS), such as: (i) PoundDrever-Hall (PDH) locking of the laser to the cavity, ${ }^{8-11}$ which gives advantages in terms of coupling efficiency, cavity injection repeatability, and increased ring-down rates, (ii) adoption of frequency-agile-rapid-scanning (FARS) schemes, ${ }^{12}$ where an optical sideband is scanned over the cavity modes with a sub-millisecond time response, (iii) locking of the laser to an ultra-stable passive cavity, which favours an efficient injection of light into the enhancement cavity, at the same time improving the short-term stability of the spectrometer. ${ }^{13,14}$ Combinations of these solutions have recently brought to exceptionally low NEA figures of $10^{-12} \mathrm{~cm}^{-1} \mathrm{~Hz}^{-0.5}$ or even beyond. ${ }^{12,15}$

In quantitative spectroscopic determinations, an enhanced or even an ultimate precision does not automatically translate into a comparable level of accuracy. In this respect, in recent years, the relevance of an adequate modeling of absorption line shapes as a prerequisite to reduce systematic errors deriving from a wrong understanding of the collisional physics has been deeply emphasized. ${ }^{16}$ Discrimination between different line shape models, as well as accurate determination of the line-centre frequency, requires a highly dense spectral sampling. Paradoxically, most techniques cited above, which have been mainly developed to reduce the NEA, do not lend themselves for dense frequency grids: locking and unlocking a PDH servo is a relatively time-consuming procedure if hundreds or thousands spectral points are targeted; FARS 
may increase the spectral scanning speed but at the price of a scarce grid of spectral points, whose separation is set by the cavity free-spectral range. Moreover, since in most cases PDH locking slaves the laser to the cavity, one needs an additional cavity stabilization to compensate for cavity drifts and to enable long averaging times. ${ }^{10} \mathrm{~A}$ few years ago, in an effort of overcoming such an issue, we proposed a comb-locked cavityring-down spectrometer in which a probe laser was tightly locked to a frequency comb, CRDS was operated in a cavityswept mode, and laser tuning was accomplished by the change of the comb repetition rate, this providing a dense and accurate frequency axis. ${ }^{17}$ On the other hand, such an approach suffered from a sub-state-of-the-art NEA of $6 \cdot 10^{-11} \mathrm{~cm}^{-1} \mathrm{~Hz}^{-0.5}$ and from a scanning time of $75 \mathrm{~s}$ for a 1500 -spectral-points spectrum, as limited by the speed of the comb repetition rate change.

This paper focuses on lay-out and performance of a deeply renewed version of that spectrometer, combining the comblocked cavity-swept CRDS concept with a frequency-agile rapid-scanning scheme. The laser remains steadily locked to a fixed repetition rate comb and spectral scanning is achieved by means of a single-side band modulator that affords a sub-ms stepping of the single sideband across adjacent cavity modes, similarly to what happens in a FARS scheme. Since the cavity is dither-locked to the sideband, once a first spectral scan is accomplished, the sideband may be shifted apart by $1-2 \mathrm{MHz}$ while remaining in the capture range of the dither-lock; from here, a second agile rapid scanning may be started in the reverse direction over the same set of modes, yet at slightly detuned cavity-modes frequencies. This enables fast interleaving of multiple spectral scans with a frequency axis fully defined by the comb. Specifically, with a ring-down rate of $1 \mathrm{kHz}$ and an empty-cavity decay time of $64 \mu \mathrm{s}$, the system covers $4.2 \mathrm{GHz}$ with a $1.3-\mathrm{MHz}$ sampling spacing (3200 points) within $6.4 \mathrm{~s}$. This time reflects the need to acquire at least two ring-down times per spectral point to compensate for the Doppler effect induced by the moving cavity mirrors. In such conditions, the root-mean-square (rms) absorption noise of blank spectra is $9.8 \cdot 10^{-11} \mathrm{~cm}^{-1}$, which translates into a noise figure of $1.7 \cdot 10^{-12} \mathrm{~cm}^{-1}$ per spectral element over $6.4 \mathrm{~s}$ after normalization against the square root number of spectral points. The precision and accuracy of the spectrometer are discussed in a fewPascal pressure range with reference to the measurements of the P12e line of the $(30012) \leftarrow(00001)$ band of $\mathrm{CO}_{2}$ at $\sim 1.57$ $\mu \mathrm{m}$. At a pressure of almost $5 \mathrm{~Pa}$, a single spectral scan is sufficient to attain a precision on the line centre-frequency of $4 \mathrm{kHz}$, which is only slightly higher than the systematic uncertainty of the spectrometer. Assuming the product between precision and acquisition time as a figure of merit, this spectrometer achieves the smallest value ever reported in a Doppler broadening regime.

\section{EXPERIMENTAL SETUP}

The experimental setup for interleaved FARS-CRDS (IFARS-CRDS) is depicted in Fig. 1. The frequency axis of the spectrometer is calibrated against a $100-\mathrm{MHz}$ Er:fibre frequency comb. Its repetition-rate is frequency locked to a low-noise quartz-oscillator that is in turn referenced to a commercial 10-MHz GPS-disciplined Rubidium clock, featuring a stability of $8 \cdot 10^{-12}$ at $1 \mathrm{~s}$. The carrier-envelope-offset frequency is extracted by a home-made $\mathrm{f}-2 \mathrm{f}$ interferometer ${ }^{18}$ and locked to the same clock by a digital proportional-integrativederivative (PID) servo, not shown in Fig. 1. A second amplified laser branch seeds a dispersion-shifted telecom fibre that provides spectral broadening from 1.4 to $1.7 \mu \mathrm{m}$ and enables a probe laser emitting in that range to be locked to the comb. Specifically, for the experiments reported here, the probe laser is an extended-cavity-diode-laser (ECDL) with wavelength tunability from 1.5 to $1.63 \mu \mathrm{m}$.

The ECDL is split in two parts by a 10:90 fibre coupler. The low-power beam is made to beat with the frequency comb

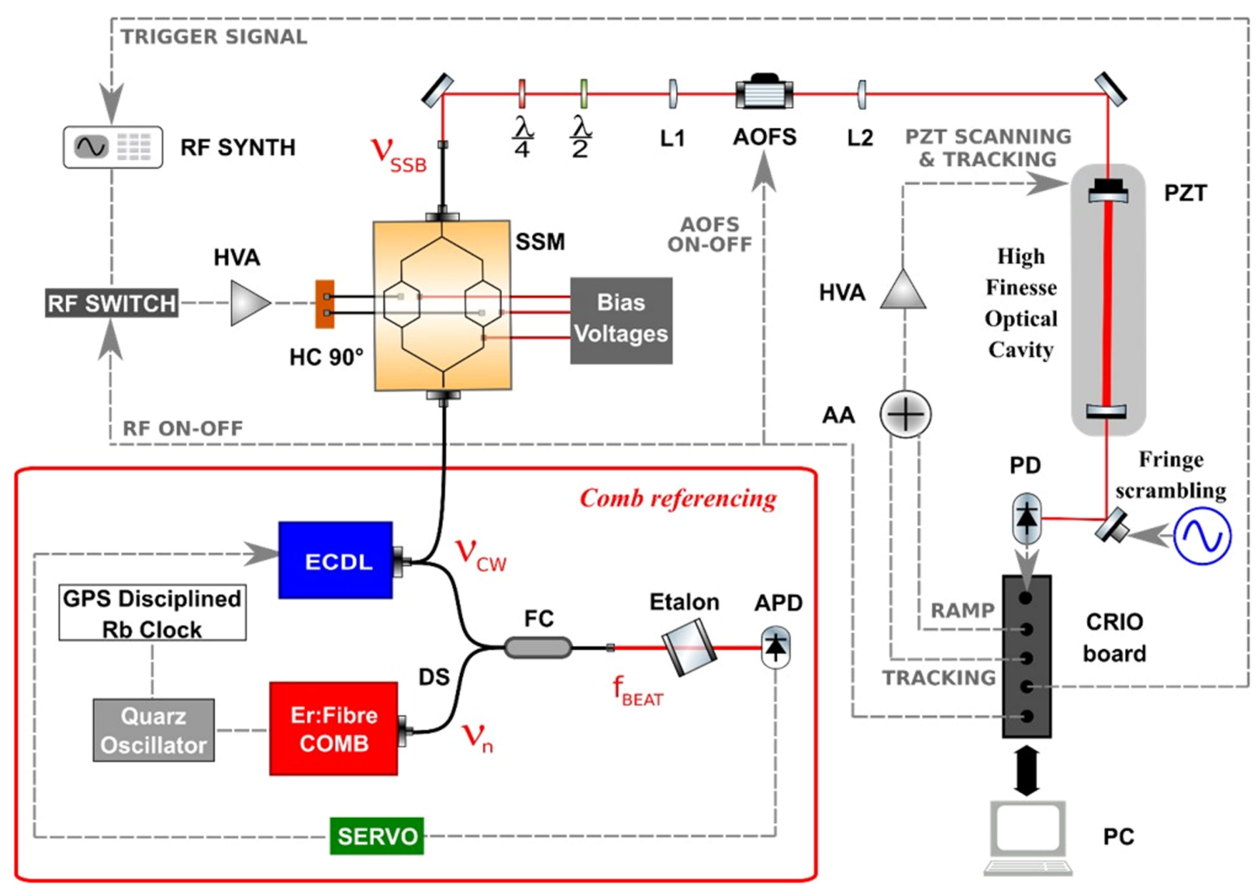

FIG. 1. Experimental setup. AOFS: acousto-optical frequency shifter; PZT: piezoelectric transducer; FC: fibre coupler; PD: photodetector; APD: avalanche photodetector; HVA: high voltage amplifier; AA: analog adder; $\mathrm{HC} 90^{\circ}$ : hybrid $90^{\circ}$ coupler; SSM: single-sideband modulator; PC: personal computer; L1, L2: lenses; DS: dispersion-shifted telecom fibre; RF SYNTH: radio-frequency synthesizer; RF SWITCH: radiofrequency electronic switch; SERVO: PID servo controller; CRIO: compact reconfigurable input/output board; $v_{\mathrm{n}}$ : $n$-th comb mode frequency; $v_{\mathrm{cw}}$ : ECDL frequency; $v_{\mathrm{SSB}}$ : single-sideband frequency; $\mathrm{f}_{\mathrm{BEAT}}$ : beat-note frequency between the comb and ECDL. Black, red, and dashed-grey lines refer to fibre, free-space laser paths, and electrical signals, respectively. 
and stabilized against it by feedback to the laser piezo modulation port (with a locking bandwidth of $4 \mathrm{kHz}$, via a commercial digital servo). The frequency stability of the beat note signal amounts to $6.5 \mathrm{kHz}$ over $10 \mathrm{~ms}$ and impacts at a level below $10^{-11}$ the repeatability of the frequency axis (at $1 \mathrm{~s}$ ). The highpower beam is sent to a single-sideband modulator ${ }^{19}$ (SSM) that affords a tuning range up to $20 \mathrm{GHz}$ and an optical conversion efficiency of $10 \%$ into the single sideband (at an RF power of $26 \mathrm{dBm}$ at the two electrodes), with a rejection of almost 50 and $35 \mathrm{~dB}$ against the carrier and the mirror sideband, respectively. The frequency shift of the sideband is set with a response time of $34 \mu$ s by a RF synthesizer (Mini circuits SSG-6400HS) operating in the $250 \mathrm{kHz}-6.4 \mathrm{GHz}$ range. In our case, the available tuning range was limited to about $4.2 \mathrm{GHz}$ by the frequency response of the RF amplifier used to drive the SSM. However, this is not an issue in the few-Pascal pressure range explored, where absorption spectra are strongly dominated by the Gaussian contribution given by Doppler broadening, with a full width at half maximum of $\sim 353 \mathrm{MHz}$ (1/12-th of the frequency scan).

The sideband generated by the SSM is used as a probe laser beam. A pair of waveplates turns its polarization into one of the polarization eigenstates of the cavity ${ }^{20}$ and this helps in reducing the fluctuations of the extracted decay rate. To enhance the extinction ratio at each ring-down event, the sideband is turned off by a combination of an acousto-optic modulator and an electronic switch placed upstream the RF ports of the SSM. The optical cavity is made up of stainless steel and it is tightened between two aluminium shells that ensure mechanical stability and high thermal conductivity. The freespectral-range of the cavity is $300 \mathrm{MHz}$, its finesse is 125000 and the input laser power is $2 \mathrm{~mW}$. Detection, data acquisition, ring-down fitting procedure, and dither-locking of the cavity to the laser do not substantially differ from what described in Ref. 17. The only improvements concern a decreased dithering amplitude, from $\pm 17 \mathrm{MHz}$ to $\pm 8.3 \mathrm{MHz}$, which reduces the mirror speed to $46 \mu \mathrm{m} / \mathrm{s}$, and the addition of a piezoelectric fringe scrambler in between the output cavity mirror and the detector to reduce parasitic etaloning effects. The piezoelectric transducer is driven by a triangular voltage ramp at a frequency of $1.4 \mathrm{~Hz}$ that produces a peak-to-peak excursion of $0.8 \mu \mathrm{m}$, squeezing fringes from $7 \cdot 10^{-11} \mathrm{~cm}^{-1}$ down to below $2 \cdot 10^{-11} \mathrm{~cm}^{-1}$.

The frequency scanning procedure is sketched in Fig. 2 . The cavity is dither-locked to the probe laser with a scanfrequency of $500 \mathrm{~Hz}$, which results in a ring-down rate of $1 \mathrm{kHz}$. Once a predetermined number of ring-down signals are acquired at a given spectral point, the SSM shifts the laser frequency from the initial cavity resonance to the adjacent one [black arrows in Fig. 2(b)], and this repeats until a first FSR-spaced spectrum is completed. During the FSR-stepped sideband tuning, the dither-locking servo just provides the bias voltage that makes the cavity resonances centred around the grid of laser frequencies. As compared to the originally proposed FARS approach, ${ }^{12}$ there is no need here for referencing the cavity against an external laser source (such as an $\mathrm{I}_{2}$-stabilized $\mathrm{He}-\mathrm{Ne}$ laser) since the cavity modes are slaved to the scanning laser. Once a first FSR-spaced spectral scan is over, the probe laser frequency is shifted apart by a small

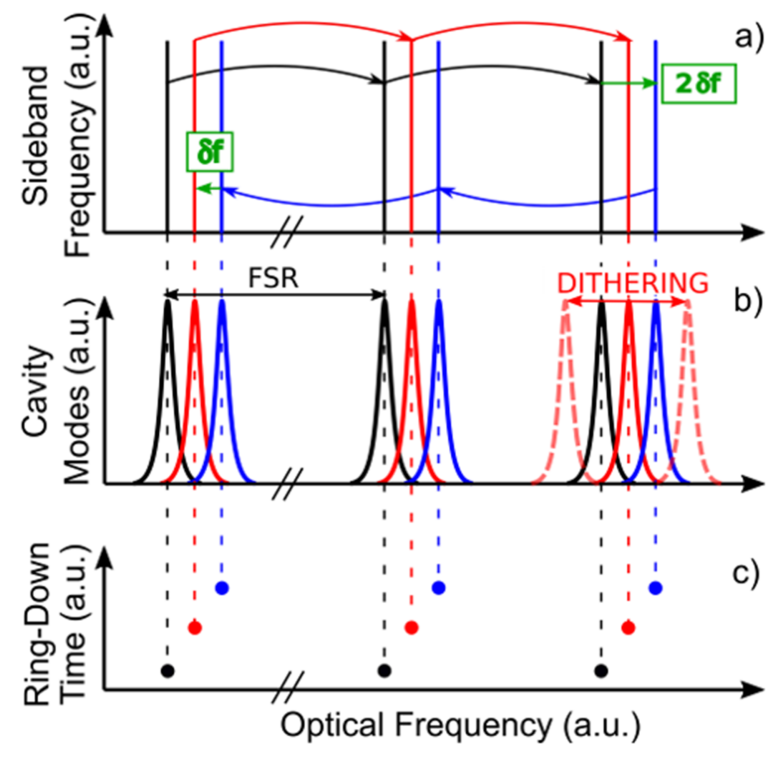

FIG. 2. (a) Set of frequencies scanned by the single sideband in a frequencyagile regime, thus in coincidence with the set of cavity modes (b). Colours represent slightly detuned sets that are interleaved with each other to define a dense frequency grid. Panel (c) gives a picture of the saw-tooth behaviour of ring-down (RD) times that may take place when the cavity-length detuning (from one set to another) induces some tiny misalignment of the intra-cavity beam: in such a case, if the mirror reflectivity does not remain perfectly uniform across its surface, a weak dependence of the ring-down time on the actual cavity length is observed.

amount ( $2 \delta \mathrm{f}$ in the figure), which makes the whole pattern of cavity modes to shift accordingly and a second spectral scan to be initiated in the reverse direction. By means of alternated scans, interleaved spectra separated by $\delta f$ are eventually achieved. As a constraint, $2 \delta$ must remain within the capture range of the dither-locking servo, i.e., within $4 \mathrm{MHz}$. Interestingly, such a scanning procedure produces a very peculiar saw-tooth behaviour for the spectral baseline. In fact, as illustrated in Fig. 2(c), it is typical to observe some slight dependence of the ring-down decay on the cavity length, which is adjusted at every intermediate spectral scan. This effect correlates with a non-uniform reflection coefficient of the mirrors and with tiny deflections of the intra-cavity optical beam that take place if the scanning mirror does not move perfectly parallel to the optical axis of the cavity. The saw-tooth pattern may occasionally lie on a more typical linear baseline if, e.g., the finesse of the cavity is not constant over the scanned frequency range.

Figure 3(a) reports a typical experimental blank spectrum that reveals the saw-tooth pattern. By fitting out this pattern, the baseline behaves as it is shown in Fig. 3(b), thus without distortions even when quite a high number $(\mathrm{N})$ of spectra are averaged together. There is no evident trace of etaloning effects, even for $\mathrm{N}=1000$, which is an indication of an effective fringe scrambling. Figure 4 better quantifies this result and reports the rms noise of blank spectra as a function of $\mathrm{N}$ (bottom horizontal scale). Actually, a departure from the expected square-root law comes gradually into play due to parasitic fringes that do not completely wash out upon averaging. The noise level remains, however, highly satisfactory and shrinks from $9.8 \cdot 10^{-11} \mathrm{~cm}^{-1}$ at $\mathrm{N}=1$ to $5 \cdot 10^{-12} \mathrm{~cm}^{-1}$ at $\mathrm{N}=1000$, which corresponds to an absorption sensitivity per 

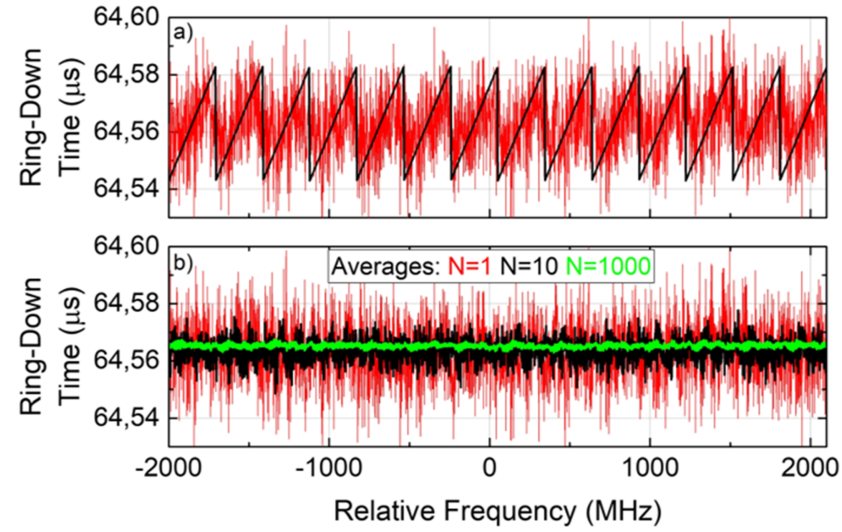

FIG. 3. (a) Typical sequence of RD-times (red) for a blank spectrum acquired in $6.4 \mathrm{~s}$, composed of 3200 points over $4.2 \mathrm{GHz}$. Black lines are a saw-tooth fitting of the experimental sequence. (b) RD-time behaviour when the sawtooth profile is fitted out: red, black, and green traces, respectively, refer to 1 , 10 , and 1000 spectra averaged together.

spectral element of $5 \cdot 10^{-12}$ and $10^{-13} \mathrm{~cm}^{-1}$, respectively, after normalization against the number of spectral points (3200). Interestingly, as highlighted by the top horizontal scale, these noise levels correspond to short acquisition times, from $6.4 \mathrm{~s}$ at $\mathrm{N}=1$ to almost 2 hours at $\mathrm{N}=1000$. These reflect the measurement of 2 ring-down times $(2 \mathrm{~ms})$ per spectral point, one for each sign of the mirror speed. In fact, due to the multiple reflections from a moving mirror during the ring-down decay, the intra-cavity field experiences a Doppler frequency shift whose sign depends on the mirror speed sign. In empty-cavity conditions, the impact of this shift on the ring-down time is negligible, but in the presence of a frequency-dependent absorption, it introduces a deformation of the absorption profile. Such deformation (see quantitative discussion and characterization in Ref. 17) remains at the second order if an equal number of ringdown times for the two speed signs are acquired and averaged together.

The spectroscopic tests were performed on the P12e line of $\mathrm{CO}_{2}$, which is a good candidate for testing the spectrometer performance since it is highly isolated from neighbouring lines. Absorption spectra have been measured on a pure $\mathrm{CO}_{2}$ sample at 36 different very low pressure values, from 0.5 to

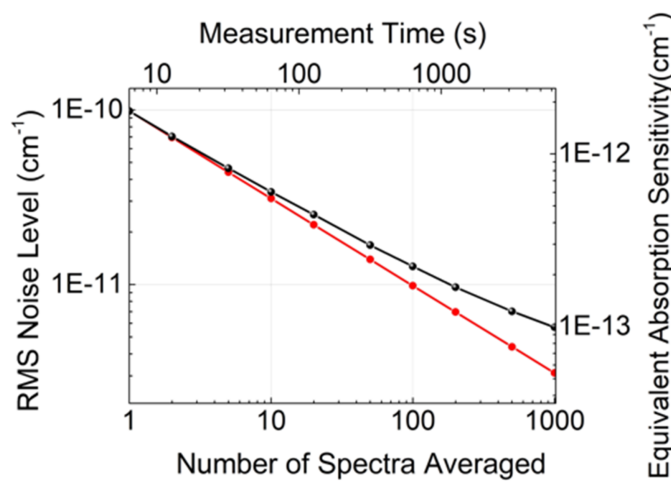

FIG. 4. Left axis: root-mean-square noise of blank spectra as a function of the number of averaged spectra (bottom scale) and of the corresponding measurement time (top scale). Right axis: absorption sensitivity per spectral element, obtained by the normalization of the noise level against the number of spectral elements (3200). The red-trace shows the ideal inverse-square-root behaviour.

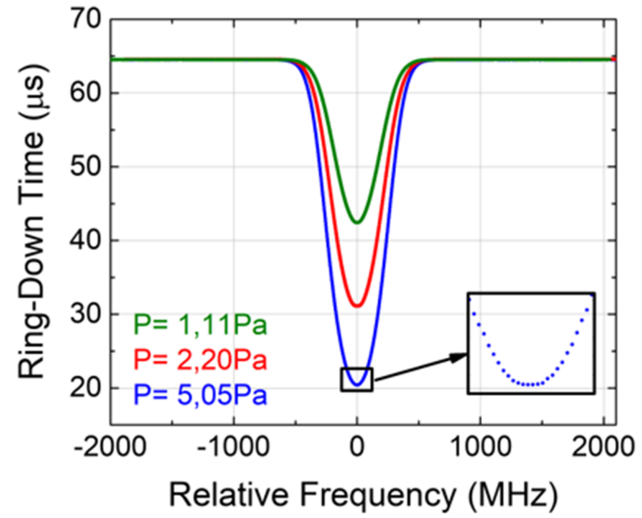

FIG. 5. Absorption profiles measured for the $(30012) \leftarrow(00$ 001) P12e line of $\mathrm{CO}_{2}$ at $\sim 1.57 \mu \mathrm{m}$ at different pressures. The measurement time scales from $4 \mathrm{~min}$ at the lower pressure to almost $10 \mathrm{~min}$ for the higher and results from the average of 35 consecutively acquired spectra, with a number of RD times per spectral point that varies from 2 on the wings of the spectrum to 16 at the line centre. The inset shows the highly dense absorption sampling.

$7 \mathrm{~Pa}$, thus in a regime where the line shape profile is dominated by Doppler broadening and exhibits a nearly Gaussian shape. In order to achieve an almost uniform signal-to-noise ratio at the wings and at the centre of the absorption spectra, the acquisition was tailored in such a way to average over a higher (yet even) number of ring-down decays at around the line centre, up to 16 . This implies one spectrum to be acquired in an interval time from 6.4 to $17 \mathrm{~s}$. At each pressure, a set of 35 consecutive spectra was acquired (within $10 \mathrm{~min}$ in the slowest case), which represents a reasonable statistical ensemble before the onset of slight drifts of the experimental conditions, mainly due to cavity leakage (at a level of $1-2 \mathrm{~Pa} / \mathrm{h}$ ). Three examples of averaged spectra are reported in Fig. 5. The lines appear continuous due to the extremely dense frequency axis, but they are indeed made of a discrete series of points, as it is evidenced by the inset of the figure; the signal-tonoise ratio exceeds 20000 already at $\sim 1 \mathrm{~Pa}$ (absorption peak: $\sim 4 \cdot 10^{-7} \mathrm{~cm}^{-1}$; noise: $2 \cdot 10^{-11} \mathrm{~cm}^{-1}$ ).

A simple Voigt profile with a fixed collisional width was used to fit the spectra, this being sufficient for the forthcoming discussion on the line-centre frequency (the application for precise line shape measurements and Doppler broadening thermometry is currently under investigation). Figure 6 reports the results of an Allan analysis performed on the line-centre values

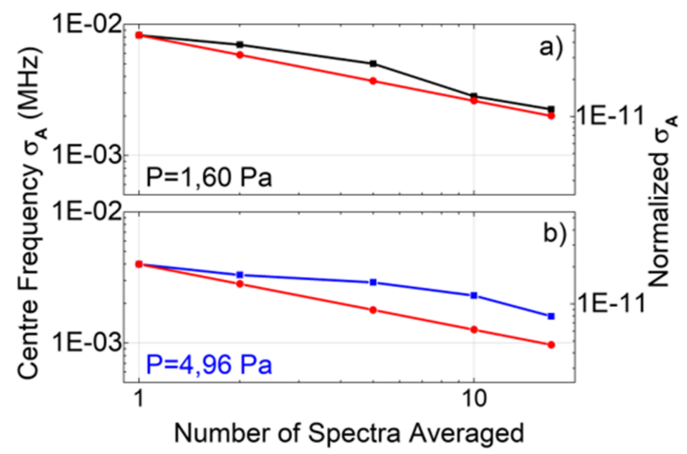

FIG. 6. Allan deviation $\sigma_{\mathrm{A}}$ (left axis) and normalized Allan deviation (right axis) calculated on the fitted line-centre values for a dataset at 1,60 Pa (a) and at $4,96 \mathrm{~Pa}(\mathrm{~b})$; the red curves represent the square root behaviour. 
retrieved from the fitting of the individual spectra of two different datasets, one at a pressure of $1.60 \mathrm{~Pa}$ [panel (a)], the other at $4.96 \mathrm{~Pa}$ [panel (b)]. At the higher pressure, the statistical uncertainty on the single line-centre determination is $4 \mathrm{kHz}$, corresponding to 2.1 parts over $10^{11}$. This is one of the highest if not the highest precision reported so far for a measurement time of $17 \mathrm{~s}$ in a Doppler-broadening regime; it is also very close to the $\mathrm{kHz}$-level afforded by sub-Doppler spectroscopy over 1 s. $^{21,22}$ The Allan plot shows that averaging over subsequent determinations (or, equivalently, extracting the line centre from an averaged profile) is beneficial for precision, but the gain given by an increasing number of averaged samples does not follow the expected inverse square-root law. This behaviour is particularly evident at higher pressures, where the signal-to-noise ratio is higher, and it is ascribed to a slight drift of the locking point of the $\mathrm{cw}$ laser with respect to the comb; this could be solved in a new setup where frequency-locking is replaced by phase-locking. Remarkably, however, a sub-kHz threshold may be broken upon a few-minutes averaging in a Doppler broadening regime.

A more stringent test on the statistical uncertainty is reported in Fig. 7, where we investigated the impact given on precision by locking-relocking procedures, namely, laser to the comb and comb (repetition rate and offset frequency) to the synthesizer, and by emptying-refilling the cavity with $\mathrm{CO}_{2}$. To this purpose, all datasets have been taken into consideration and a mean-value for the line centre at each pressure was retrieved and plotted in Fig. 7. A weighted fit of the mean frequencies with a linear function returns a type A (statistical) error of $1.6 \mathrm{kHz}$ for the zero-pressure value, thus in reasonably good agreement with the $\mathrm{kHz}-$ level estimated by the Allan analysis of the single datasets. The overall reproducibility of the spectrometer is thus highly satisfactory and nearly a factor of 10 better than our previous implementation. ${ }^{17}$ The self-induced pressure-shift coefficient retrieved from the linear fitting is $-2.80 \pm 0.42 \mathrm{kHz} / \mathrm{Pa}$ : it is within $3 \sigma$ in agreement with data from Devi et al. ${ }^{23}(-1.49 \pm 0.02 \mathrm{kHz} / \mathrm{Pa})$, but the two pressure regimes differ by about 4 orders of magnitude.

The linear fit in Fig. 7 allows a frequency of $190008170.1805 \pm 0.0021 \mathrm{MHz}$ to be extrapolated at zero

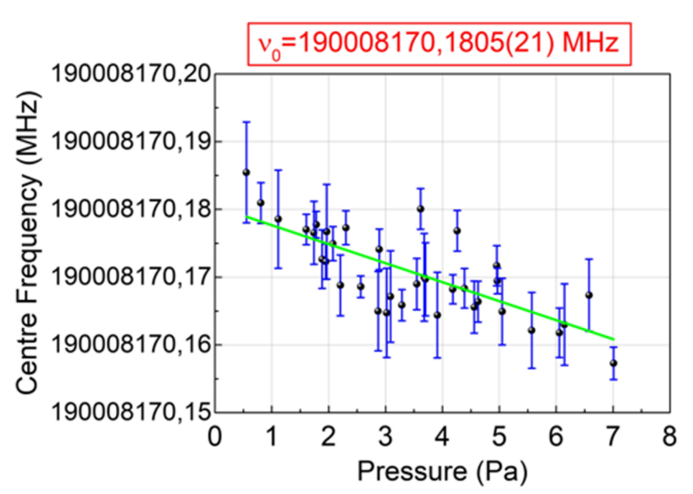

FIG. 7. Mean line-centre values at different pressures and corresponding weighted linear fit (green line) to extrapolate the centre frequency at zero pressure. The error bars are retrieved from the Allan deviation analysis at $\mathrm{N}$ $=17$.
TABLE I. Absolute frequencies in this work and in the literature for the (30 $012) \leftarrow(00001)$ P12e line of $\mathrm{CO}_{2}$ (second column), uncertainty level (third column) and comparison against this work (this work minus other references, fourth column).

\begin{tabular}{lccc}
\hline \hline P12e & Centre frequency (MHz) & Uncertainty $(\mathrm{kHz})$ & Deviation $(\mathrm{kHz})$ \\
\hline This work & 190008170.1805 & 2.1 & $\ldots$ \\
HITRAN $^{24}$ & 190008171.960 & $300-3000$ & -1780 \\
Devi et al. $^{23}$ & 190008170.850 & 600 & -670 \\
Long et al. $^{25}$ & 190008170.101 & 30 & 79 \\
\hline \hline
\end{tabular}

pressure. The uncertainty is calculated as the quadrature addition of a $1.6-\mathrm{kHz}$ type A error and a $1.4-\mathrm{kHz}$ type B (systematic) error: the later reflects a number of contributions thoroughly discussed in Ref. 17, which include the stability of the GPS-based frequency standard $(0.6 \mathrm{kHz})$, the accuracy of the absolute pressure gauge $(0.4 \mathrm{kHz})$, the cavity leakage $(0.16 \mathrm{kHz})$, and the effect of the Doppler shift given by the moving mirror (here more prudentially considered at a $2 \sigma$ level, i.e., $1.2 \mathrm{kHz}$ ). Table I compares the line-centre frequency retrieved in this work with data from the literature for the same line, specifically from HITRAN, ${ }^{24}$ Devi et al. ${ }^{23}$ who exploited a state-of-the-art Fourier-transform measurement, and Long et al. ${ }^{25}$ who made use of a FARS CRDS approach. With respect to HITRAN ${ }^{24}$ and Devi et al., ${ }^{23}$ the agreement is within $1 \sigma$, with a departure of 1.78 and $0.67 \mathrm{MHz}$, respectively. As compared to the comb-calibrated data by Long et al. ${ }^{25}$ the discrepancy decreases down to $79 \mathrm{kHz}$, which corresponds to $\sim 2.5 \sigma$ of their declared uncertainty (approximately one order of magnitude larger than that provided here).

\section{CONCLUSIONS}

In conclusion, we have introduced interleaved FARS CRDS as a powerful approach to conjugate high sensitivity, fast acquisition time, and a very dense frequency grid. With the addition of an optical frequency comb, this represents an effective way to considerably enhance the precision and the accuracy of line-centre frequency determinations over short measurement times, down to $4 \mathrm{kHz}$ over less than $20 \mathrm{~s}$, which is to our knowledge the best result obtained without recurring to sub-Doppler regimes. This is very important for the measurement of weak absorption lines of atmospheric interest or for more fundamental research on electric-quadrupole transitions in molecular hydrogen or deuterium. The approach presented is especially appropriate for accurate modeling of the absorption line shapes, as facilitated by the highly dense spectral sampling. Additionally, extremely fast acquisition rate allows one to avoid systematic effects introduced by the sample composition or by environmental changes in the system.

\section{ACKNOWLEDGMENTS}

The authors acknowledge financial support from "Extreme Light Infrastructure," Italy.

${ }^{1}$ M. L. Niu, E. J. Salumbides, G. D. Dickenson, K. S. E. Eikema, and W. Ubachs, "Precision spectroscopy of the $\mathrm{X}^{1} \Sigma_{\mathrm{g}}{ }^{+}, \mathrm{v}=0 \rightarrow 1(\mathrm{~J}=0-2)$ rovibrational splittings in $\mathrm{H}_{2}, \mathrm{HD}$ and $\mathrm{D}_{2}$," J. Mol. Spectrosc. 300, 44-54 (2014). 
${ }^{2}$ C. Daussy, M. Guinet, A. Amy-Klein, K. Djerroud, Y. Hermier, S. Briaudeau, C. J. Bordé, and C. Chardonnet, "Direct determination of the Boltzmann constant by an optical method," Phys. Rev. Lett. 98, 250801 (2007).

${ }^{3}$ L. Moretti, A. Castrillo, E. Fasci, M. D. De Vizia, G. Casa, G. Galzerano, A. Merlone, P. Laporta, and L. Gianfrani, "Determination of the Boltzmann constant by means of precision measurements of $\mathrm{H}_{2}{ }^{18} \mathrm{O}$ line shapes at 1:39 $\mu$ m," Phys. Rev. Lett. 111, 060803 (2013).

${ }^{4}$ A. Campargue, S. Kassi, K. Pachucki, and J. Komasa, "The absorption spectrum of $\mathrm{H}_{2}$ : CRDS measurements of the (2-0) band, review of the literature data and accurate $a b$-initio line list up to $35000 \mathrm{~cm}^{-1}$," Phys. Chem. Chem. Phys. 14, 802 (2012).

${ }^{5}$ S. Kassi, A. Campargue K. Pachucki, and J. Komasa, "The absorption spectrum of $\mathrm{D}_{2}$ : Ultrasensitive cavity ring down spectroscopy of the (2-0) band near $1.7 \mu \mathrm{m}$ and accurate ab initio line list up to $24000 \mathrm{~cm}^{-1}$," J. Chem. Phys. 136, 184309 (2012).

${ }^{6}$ J. Burkart, T. Sala, D. Romanini, M. Marangoni, A. Campargue, and S. Kassi, "Communication: Saturated $\mathrm{CO}_{2}$ absorption near $1.6 \mu \mathrm{m}$ for kilohertz-accuracy transition frequencies," J. Chem. Phys. 142, 191103 (2015).

${ }^{7}$ C.-F. Cheng, J. Wang, Y. R Sun, Y. Tan, P. Kang, and S.-M. Hu, "Doppler broadening thermometry based on cavity ring-down spectroscopy," Metrologia 52, S385-S393 (2015).

${ }^{8}$ T. G. Spence, C. C. Harb, B. A. Paldus, R. N. Zare, B. Willke, and R. L. Byer, "A laser-locked cavity ring-down spectrometer employing an analog detection scheme,” Rev. Sci. Instrum. 71, 347-353 (2000).

${ }^{9}$ R. Z. Martínez, M. Metsälä, O. Vaittinen, T. Lantta, and L. Halonen, "Laserlocked, high-repetition-rate cavity ringdown spectrometer," J. Opt. Soc. Am. B 23, 727-740 (2006).

${ }^{10}$ A. Cygan, D. Lisak, P. Masłowski, K. Bielska, S. Wójtewicz, J. Domysławska, R. S. Trawinski, R. Ciuryło, H. Abe, and J. T. Hodges, "Pound-Drever-Hall-locked, frequency-stabilized cavity ring-down spectrometer," Rev. Sci. Instrum. 82, 063107 (2011).

${ }^{11}$ K. Bielska, S. Wójtewicz, P. Morzyǹski, P. Ablewski, A. Cygan, M. Bober, J. Domysławska, M. Zawada, R. Ciuryło, P. Masłowski, and D. Lisak, "Absolute frequency determination of molecular transition in the Doppler regime at kHz level of accuracy," J. Quant. Spectrosc. Radiat. Transfer 201, $156-160$ (2017).

${ }^{12}$ G.-W. Truong, K. O. Douglass, S. E. Maxwell, R. D. van Zee, D. F. Plusquellic, J. T. Hodges, and D. A. Long, "Frequency-agile, rapid scanning spectroscopy," Nat. Photonics 7, 532 (2013).

${ }^{13}$ A. Cygan, D. Lisak, P. Morzyński, M. Bober, M. Zawada, E. Pazderski, and R. Ciuryło, "Cavity mode-width spectroscopy with widely tunable ultra narrow laser," Opt. Express 21, 29744-29754 (2013).

${ }^{14}$ J. Burkart, D. Romanini, and S. Kassi, "Optical feedback frequency stabilized cavity ring-down spectroscopy,” Opt. Lett. 39, 4695-4698 (2014).
${ }^{15}$ D. A. Long, A. J. Fleisher, S. Wójtewicz, and T. Hodges, "Quantumnoise-limited cavity ring-down spectroscopy,” Appl. Phys. B 115, 149-153 (2014).

${ }^{16}$ N. H. Ngo, D. Lisak, H. Tran, and J. M. Hartmann, "An isolated lineshape model to go beyond the Voigt profile in spectroscopic databases and radiative transfer codes," J. Quant. Spectrosc. Radiat. Transfer 129, 89-100 (2013).

${ }^{17}$ D. Gatti, T. Sala, R. Gotti, L. Cocola, L. Poletto, M. Prevedelli, P. Laporta, and M. Marangoni, "Comb-locked cavity ring-down spectrometer," J. Chem. Phys. 142, 074201 (2015).

${ }^{18}$ F. Tauser, A. Leitenstorfer, and W. Zinth, "Amplified femtosecond pulses from an Er:fiber system: Nonlinear pulse shortening and self-referencing detection of the carrier-envelope phase evolution," Opt. Express 11, 594600 (2003).

${ }^{19}$ J. Burkart, T. Sala, S. Kassi, D. Romanini, and M. Marangoni, "Optical phase cloning by an integrated dual-parallel Mach-Zehnder modulator," Opt. Lett. 40, 816-819 (2015).

${ }^{20} \mathrm{H}$. Huang and K. K. Lehmann, "Effects of linear birefringence and polarization-dependent loss of supermirrors in cavity ring-down spectroscopy," Appl. Opt. 47, 3817-3827 (2008).

${ }^{21}$ S. Twagirayezu, M. J. Cich, T. J. Sears, C. P. McRaven, and G. E. Hall, "Frequency-comb referenced spectroscopy of $v_{4}$ - and $v_{5}$-excited hot bands in the $1.5 \mu \mathrm{m}$ spectrum of $\mathrm{C}_{2} \mathrm{H}_{2}$," J. Mol. Spectrosc. 316, 64-71 (2015).

${ }^{22}$ D. Gatti, R. Gotti, A. Gambetta, M. Belmonte, G. Galzerano, P. Laporta, and M. Marangoni, "Comb-locked Lamb-dip spectrometer," Sci. Rep. 6, 27183 (2016).

${ }^{23}$ V. M. Devi, D. C. Benner, L. R. Brown, C. E. Miller, and R. A. Toth, "Line mixing and speed dependence in $\mathrm{CO}_{2}$ at $6348 \mathrm{~cm}^{-1}$ : Positions, intensities, and air- and self-broadening derived with constrained multispectrum analysis," J. Mol. Spectrosc. 242, 90-117 (2007).

${ }^{24}$ L. S. Rothman, I. E. Gordon, Y. Babikov, A. Barbe, D. Chris Benner, P. F. Bernath, M. Birk, L. Bizzocchi, V. Boudon, L. R. Brown, A. Campargue, K. Chance, E. A. Cohen, L. H. Coudert, V. M. Devi, B. J. Drouin, A. Fayt, J.-M. Flaud, R. R. Gamache, J. J. Harrison, J.-M. Hartmann, C. Hill, J. T. Hodges, D. Jacquemart, A. Jolly, J. Lamouroux, R. J. Le Roy, G. Li, D. A. Long, O. M. Lyulin, C. J. Mackie, S. T. Massie, S. Mikhailenko, H. S. P. Müller, O. V. Naumenko, A. V. Nikitin, J. Orphal, V. Perevalov, A. Perrin, E. R. Polovtseva, C. Richard, M. A. H. Smith, E. Starikova, K. Sung, S. Tashkun, J. Tennyson, G. C. Toon Vl, G. Tyuterev, and G. Wagner, J. Quant. Spectrosc. Radiat. Transfer 130, 4 (2013).

${ }^{25}$ D. A. Long, S. Wójtewicz, C. E. Miller, and J. T. Hodges, "Frequencyagile, rapid scanning cavity ring-down spectroscopy(FARS-CRDS) measurements of the (30012)-(00001) near-infrared carbon dioxide band," J. Quant. Spectrosc. Radiat. Transfer 161, 35-40 (2015). 